\title{
Estratégias utilizadas na prática dos cuidados paliativos em terapia intensiva neopediátrica
}

\author{
Strategies used in the practice of palliative care in neopediatric intensive therapy \\ Estrategias utilizadas en la prática de cuidados paliativos en terapia intensiva neopediátrica
}

Recebido: 14/07/2021 | Revisado: 22/07/2021 | Aceito: 15/08/2021 | Publicado: 17/08/2021

\author{
Alexandre Ernesto Silva \\ ORCID: https://orcid.org/0000-0001-9988-144X \\ Universidade Federal de São João Del Rei, Brasil \\ E-mail: alexandresilva@ufsj.edu.br \\ Rosimeire de Paula Silva \\ ORCID: https://orcid.org/0000-0002-3403-8862 \\ Universidade Federal de São João Del Rei, Brasil \\ E-mail: rosimeiresgp@gmail.com \\ Danyelle Maria Silva \\ ORCID: https://orcid.org/0000-0002-4465-4976 \\ Universidade Federal de São João Del Rei, Brasil \\ E-mail: danyellesilvamaria@gmail.com \\ Hellen Carolina Ferreira Moraes \\ ORCID: https://orcid.org/0000-0001-9859-1977 \\ Universidade Estadual de Minas Gerais, Brasil \\ E-mail: hellenfmoraes@hotmail.com \\ Carolina Eloi Miranda \\ ORCID: https://orcid.org/0000-0002-7641-6673 \\ Universidade Federal de São João Del Rei, Brasil \\ E-mail: carolina.miranda@cssjd.org.br \\ Jaqueline Layane da Silva Inácio \\ ORCID: https://orcid.org/0000-0002-9035-9847 \\ Universidade Federal de São João Del Rei, Brasil \\ E-mail: jacinacio03@gmail.com \\ Stefãne Amorim Ribeiro \\ ORCID: https://orcid.org/0000-0001-9702-1913 \\ Faculdade Asa de Brumadinho, Brasil \\ E-mail:stefanearibeiro@yahoo.com.br \\ Thayane Vieira Carvalho \\ ORCID: https://orcid.org/0000-0001-8644-8954 \\ Clínica Auge, Brasil \\ E-mail: thayanevieira100@yahoo.com.br
}

\begin{abstract}
Resumo
Quando se pensa no curso natural da vida imagina-se que o ser humano nasce, cresce, envelhece e só após isso passa pelo processo de morte. Em uma unidade de tratamento intensivo neonatal e pediátrica - UTI-Neopediátrica, o percurso da vida pode se mostrar encurtado, e o fim da vida pode se tornar um fato real ao recém-nascido/criança. Ao analisar as condutas tomadas frente ao paciente, tratando-se da enfermagem, a abordagem paliativa segue uma linha macro de cuidado, se restringindo ao cumprimento de prescrições médicas e realização de procedimentos. Diante da importância da abordagem dos cuidados paliativos, levantamos a seguinte pergunta: Quais são as estratégias utilizadas por médicos e enfermeiros na elegibilidade e assistência paliativa aos pacientes internados em uma UTI neopediátrica, e aos seus familiares? Os artigos foram selecionados e organizados seguindo a estratégia PICO. Após realizar a delimitação da literatura disponível, uma análise criteriosa foi feita para que as temáticas mais relevantes dos artigos pudessem ser organizadas. Diversos obstáculos afetam diretamente a assistência como a formação, a complexidade dos procedimentos, a falta de privacidade com paciente e familiares, despreparo profissional, dentre outros. Por fim, conclui-se que a revisão de literatura conseguiu reunir um conteúdo consistente e muito proveitoso, contribuindo para o enriquecimento do conhecimento acerca dos Cuidados Paliativos e como os profissionais médicos e da enfermagem conseguem abordar cuidados paliativos na uti neonatal buscando sempre oferecer a melhor assistência a seus pacientes e familiares apesar de todos os desafios encontrados na rotina do serviço.
\end{abstract}

Palavras-chave: Cuidados paliativos; Neonatologia; Pediatria; Unidade de Terapia Intensiva. 


\begin{abstract}
When one thinks about the natural course of life, one imagines that the human being is born, grows, grows old and only then goes through the process of death. In a neonatal and pediatric intensive care unit - Neopediatric ICU, the course of life can be shortened, and the end of life can become a real fact for the newborn/child. When analyzing the actions taken towards the patient, in the case of nursing, the palliative approach follows a macro line of care, restricted to compliance with medical prescriptions and carrying out procedures. Given the importance of the palliative care approach, we raise the following question: What are the strategies used by physicians and nurses in the eligibility and palliative care of patients hospitalized in a neopediatric ICU, and their families? The articles were selected and organized following the PICO strategy. After delimiting the available literature, a careful analysis was carried out so that the most relevant topics in the articles could be organized. Several obstacles directly affect care, such as training, complexity of procedures, lack of privacy with patients and family members, professional unpreparedness, among others. Finally, it is concluded that the literature review managed to gather a consistent and very useful content, contributing to the enrichment of knowledge about Palliative Care and how medical and nursing professionals are able to approach palliative care in the neonatal ICU, always seeking to offer the best assistance to their patients and families despite all the challenges encountered in the routine of the service.
\end{abstract}

Keywords: Palliative care; Neonatology; Pediatrics; Intensive Care Unit.

\title{
Resumen
}

Cuando se piensa en el curso natural de la vida, se imagina que el ser humano nace, crece, envejece y sólo entonces pasa por el proceso de la muerte. En una unidad de cuidados intensivos neonatales y pediátricos, la UCI neopediátrica, el curso de la vida se puede acortar y el final de la vida puede convertirse en un hecho real para el recién nacido / niño. Al analizar las acciones realizadas hacia el paciente, en el caso de la enfermería, el abordaje paliativo sigue una macrolínea de cuidados, restringida al cumplimiento de las prescripciones médicas y realización de procedimientos. Dada la importancia del enfoque de cuidados paliativos, nos planteamos la siguiente pregunta: ¿Cuáles son las estrategias que utilizan los médicos y enfermeras en la elegibilidad y cuidados paliativos de los pacientes hospitalizados en una UCI neopediátrica y sus familias? Los artículos fueron seleccionados y organizados siguiendo la estrategia PICO. Luego de delimitar la literatura disponible, se realizó un análisis cuidadoso para que se pudieran organizar los temas más relevantes de los artículos. Varios obstáculos afectan directamente la atención, como la capacitación, la complejidad de los procedimientos, la falta de privacidad con los pacientes y familiares, la falta de preparación profesional, entre otros. Finalmente, se concluye que la revisión de la literatura logró recoger un contenido consistente y muy útil, contribuyendo al enriquecimiento del conocimiento sobre Cuidados Paliativos y cómo los profesionales médicos y de enfermería son capaces de abordar los cuidados paliativos en la UCI neonatal, buscando siempre ofrecer el la mejor asistencia a sus pacientes y familiares a pesar de todos los desafíos encontrados en la rutina del servicio.

Palabras clave: Cuidados paliativos; Neonatologia; Pediatria; Unidad de Terapia Intensiva.

\section{Introdução}

Quando se pensa no curso natural da vida imagina-se que o ser humano nasce, cresce, envelhece e só após isso passa pelo processo de morte. Em uma unidade de tratamento intensivo neonatal e pediátrica - UTI-Neopediátrica, o percurso da vida pode se mostrar encurtado, e o fim da vida pode se tornar um fato real ao recém-nascido/criança. Lidar com o processo de morte em uma UTI-Neopediátrica é uma grande dificuldade para a equipe profissional, pois demanda do mesmo conhecimento para manejar de forma tecnicamente e humanamente adequada essa situação tão delicada (Almeida \& Moraes, 2016).

O destaque dos cuidados paliativos neonatal no Brasil iniciou-se pelo Centro de Atenção Integral à Saúde da Mulher (CAISM) em 2002, onde a equipe de neonatologia passou a se reunir semanalmente para a discussão de casos, incorporação de protocolos, reuniões com familiares de bebês internados e revisão teórica dos casos. Em virtude dos fatos, a equipe apresentou os primeiros princípios norteadores para os Cuidados Paliativos Neonatal. Essa proposta foi integrada às rotinas com o intuito de melhorias nas linhas de cuidados e de articular o envolvimento da equipe multiprofissional (World Health Organization, 2018).

Segundo a Organização Mundial da Saúde (OMS), os cuidados paliativos consistem na aplicação de ações desenvolvidas por uma equipe multiprofissional que busque promover qualidade de vida ao paciente e sua família diante de uma doença ou condição que ameace a continuidade da vida, por meio do alívio do sofrimento físico, psicológico, espiritual e social. Não diferente disso, os cuidados paliativos na neopediatria seguem os mesmos princípios através da colaboração 
interdisciplinar que busca melhorar a qualidade de vida de crianças com risco de morte, bem como o acolhimento e acompanhamento de seus familiares e se concentra na prevenção e no alívio do sofrimento (Côté, Payot \& Gaucher, 2020).

De acordo com a Academia Americana de Pediatria, o cuidado paliativo pediátrico deve ser um modelo integral que consiga garantir a dignidade dos pacientes bem como de suas famílias, acesso a um serviço compassivo e capacitado, existência de um serviço que ofereça suporte aos profissionais de saúde, melhora do suporte social e profissional para os cuidados paliativos em pediatria e incentivo a pesquisas e estudos que abordem essa área. Diante desses aspectos, a Sociedade Brasileira de Pediatria criou os princípios dos cuidados paliativos em pediatria, uma vez que está bem definido que não se pode extrapolar para o paciente pediátrico todos os conceitos e estratégias utilizadas em adultos. Estes princípios podem nortear a abordagem dos cuidados paliativos às crianças e seus familiares (SBP, 2017).

Assim, as orientações dos cuidados deverão ser bem esclarecidas para a família, visando o estabelecimento de uma parceria que promova uma abertura para trocas significativas, favorecendo a compreensão e o manejo de cada desafio. A assistência deve se concentrar em medidas que busquem minimizar sintomas e garantir conforto ao paciente e segurança à família. Serão elegíveis para cuidados paliativos crianças que apresentem doenças crônicas, em condições de terminalidade ou que ameacem a sobrevida. O acompanhamento deve ser realizado durante todo o tempo necessário e a abordagem multiprofissional deverá ser encorajada, podendo esta ser feita em âmbito hospitalar ou não, e não se restringindo a pacientes com expectativa de sobrevida breve (SBP, 2017).

A morte em neonatologia é algo pouco discutida, visto que se trata de um momento que exige importante e difícil compreensão. Isso porque na realidade, em terapia intensiva neopediátrica a abordagem profissional em muitos casos se baseia em tentar a todo custo buscar uma medida curativa para a condição crítica do paciente, o que pode não ser possível em todas as situações. Dentro dos objetivos dos cuidados paliativos todos os recém-nascidos e crianças em condições que ameacem a vida são beneficiados dos cuidados paliativos, havendo possibilidade de cura ou não, em relação a controle de sintomas de alívio de sofrimentos, em alguns casos devem ser inseridos os cuidados paliativos como abordagem multiprofissional de forma exclusiva para com o recém-nascido e seus familiares, sendo esses os casos onde não há mais possibilidade de medidas curativas. Os desafios da equipe de enfermagem no contexto da unidade de terapia intensiva (UTI) Neopediátrica se dá por meio de barreiras, como a falta de diálogo entre a equipe de saúde, a impossibilidade de opinar nas decisões de final de vida e a falta de preparo para lidar com essas situações, isso se mostra como entraves que se refletem nas relações do cuidado (World Health Organization, 2017).

Para os profissionais que trabalham em pediatria, estudos sustentam que a capacitação em cuidados paliativos ainda é muito escassa, o que pode deixar os pacientes e seus familiares carentes desse tipo de assistência quando necessário. A falta de capacitação ou a capacitação inadequada acabam por criar um estigma dentro das UTI's neopediátricas, expondo que a abordagem paliativa é sinônimo de falha no papel de promoção da saúde desenvolvido pelos profissionais, logo essa ação é deixada de lado ou é tomada tardiamente, sendo restrita aos profissionais médicos (Henner \& Boss, 2017; Zimmermann et al., 2018).

Schwartz e Chalupka (2019) conclui em sua pesquisa que as dificuldades para a implementação das ações dos cuidados paliativos pediátricos se assemelham a nível internacional. Estados Unidos, Reino Unido, Canadá e Austrália apresentaram uma demanda voltada para a capacitação dos profissionais que lidam diretamente na assistência, para identificar quando o encaminhamento para cuidados paliativos pediátrico (CPP) é indicado para uma criança. Dentre as principais dificuldades para se implementar os cuidados há um déficit na habilidade de comunicação entre profissional de saúde e família do paciente. Quando comparado com o estudo desenvolvido por Silva, Valença e Germano (2010) é possível perceber através dos discursos analisados pela autora que esse contexto também se aplica à realidade vivenciada nas UTI's neonatais localizadas em hospitais brasileiros. Em um panorama geral temos que os fatores dificultadores que mais são descritos nos 
estudos se voltam para o preparo da equipe multiprofissional para realizar esse tipo de assistência (Schwartz \& Chalupka, 2020).

Ao analisar as condutas tomadas frente ao paciente, tratando-se da enfermagem, a abordagem paliativa segue uma linha macro de cuidado, se restringindo ao cumprimento de prescrições médicas e realização de procedimentos. Muitas vezes o papel de estabelecer o vínculo com a família, promover e auxiliar em momentos de grande vulnerabilidade não parece prioritário. No cuidado paliativo pediátrico a equipe de enfermagem terá um fator determinante para a garantia de qualidade na assistência e, por isso é de extrema importância o seu preparo para lidar com as famílias e com todos os profissionais da equipe multidisciplinar. Já ao analisar a equipe médica nota-se que há uma dificuldade na identificação da necessidade dos cuidados paliativos e, por isso, o encaminhamento do paciente é realizado de forma tardia. A assistência médica acaba por ser centrada na resolução da doença, sendo voltado para medidas curativas que muitas vezes são realizadas sem que haja de fato um benefício para o paciente (Silva et al., 2017).

Diante da importância da abordagem dos cuidados paliativos como promotor da dignidade humana nas diferentes etapas da vida, e demonstrada incipiência de estudos atuais nesta temática voltadas aos recém-nascidos e crianças, e na fragilidade na formação de profissionais de saúde que trabalham neste cenário, emerge a seguinte pergunta: Quais são as estratégias estratégias utilizadas por médicos e enfermeiros na elegibilidade e assistência paliativa aos pacientes internados em uma UTI neopediátrica, e aos seus familiares?

\section{Metodologia}

Baseada na análise ampla e sistemática de artigos científicos a revisão integrativa da literatura pode ser compreendida como um método de pesquisa que permite uma profunda compreensão acerca de uma temática e como está a perspectiva dos autores perante a mesma. A partir da integração de opiniões e conceitos de pesquisas já existentes a revisão bibliográfica do tipo integrativa permite que seja realizada uma síntese e análise do conhecimento científico já existente (Souza, Silva \& Carvalho, 2010).

Para o presente estudo foram analisados artigos publicados no intervalo de tempo de 2016 a 2021 e que tenham o texto completo disponibilizado. Foram utilizadas para a pesquisa as bases de dados Biblioteca Virtual em Saúde (BVS), que incluem: Base de Dados de Enfermagem (Bdenf), Literatura Latino-Americana e do Caribe em Ciências da Saúde (Lilacs) e coleção Scientific Electronic Library online (Scielo); e também base de dados Medline, através da PubMed - National Library Of Medicine National Institutes Of Health. O refinamento da pesquisa foi feito utilizando os descritores Cuidados paliativos, Neonatologia, Pediatria e Unidade de terapia intensiva. Artigos que não tenham caráter científico ou que não se enquadrem na temática do estudo foram excluídos. Garantindo a consideração ética todos os autores terão seus estudos rigorosamente referenciados.

Os artigos foram selecionados e organizados seguindo a estratégia PICO, que é utilizada para a construção de temas de pesquisa. O resumo dos dados encontrados através dessa estratégia é demonstrado no Quadro 1 a seguir, onde se descreve o tipo de paciente utilizado no estudo, a intervenção realizada e o tipo de tratamento indicado. 
Research, Society and Development, v. 10, n. 10, e506101018277, 2021

(CC BY 4.0) | ISSN 2525-3409 | DOI: http://dx.doi.org/10.33448/rsd-v10i10.18277

Quadro 1 - Descrição da estratégia Pico - Belo Horizonte, Minas Gerais, 2021

\begin{tabular}{|c|c|c|}
\hline ACRÔNIMOS & DESCRIÇÃO & ANÁLISE \\
\hline $\mathrm{P}$ & Paciente & $\begin{array}{c}\text { Pacientes em terapia intensiva } \\
\text { neopediátrica. }\end{array}$ \\
\hline $\mathrm{I}$ & Intervenção ou indicador & Estratégias de cuidados paliativos. \\
\hline $\mathrm{C}$ & Comparação ou controle & Não se aplica. \\
\hline $\mathrm{O}$ & Outcomes - desfecho & Eficácia dos cuidados paliativos. \\
\hline
\end{tabular}

Fonte: Santos, Pimenta \& Nobre (2007).

\section{Resultados e Discussão}

Através da leitura dos artigos buscou-se analisar os seguintes aspectos: área do conhecimento, variáveis estudadas, método e relevância da pesquisa. Feito o delineamento dos critérios de inclusão e exclusão dos artigos, foram selecionadas inicialmente 39 produções nas quais os títulos se enquadraram dentro dos critérios de inclusão, essa seleção após leitura minuciosa dos textos completos se afunilou para 10 estudos. Na tabela a seguir estão dispostos os artigos selecionados.

Após realizar a delimitação da literatura disponível, uma análise criteriosa foi feita para que as temáticas mais relevantes dos artigos pudessem ser organizadas, visando a coleta das informações necessárias para responder à pergunta da pesquisa. Ao analisar as obras selecionadas é possível observar que a maioria utilizou um método qualitativo para descrição e análise dos dados (90\% da amostra). Em relação às bases de dados a quantidade de artigos presentes na MEDLINE (PUBMED) se mostrou significativamente maior (60\%) quando comparada às outras. As publicações que abordam o tema são majoritariamente internacionais e em inglês, sendo selecionados apenas 3 artigos brasileiros (30\%).

Os resultados da busca estão dispostos no Quadro 2 a seguir que está categorizada de acordo com as variáveis encontradas nos artigos selecionados. 
Research, Society and Development, v. 10, n. 10, e506101018277, 2021

(CC BY 4.0) | ISSN 2525-3409 | DOI: http://dx.doi.org/10.33448/rsd-v10i10.18277

Quadro 2: Categorização dos estudos. - Belo Horizonte, Minas Gerais, 2021

\begin{tabular}{|c|c|c|}
\hline Variáveis & $\mathrm{N}$ amostral & $\%$ \\
\hline Artigos selecionados & 10 & 100 \\
\hline \multicolumn{3}{|c|}{ Tipo de estudo } \\
\hline Quantitativo & 1 & 10 \\
\hline Qualitativo & 9 & 90 \\
\hline \multicolumn{3}{|c|}{ Ano de publicação } \\
\hline 2016 & 0 & 0 \\
\hline 2017 & 3 & 30 \\
\hline 2018 & 1 & 10 \\
\hline 2019 & 3 & 30 \\
\hline 2020 & 2 & 20 \\
\hline 2021 & 1 & 10 \\
\hline \multicolumn{3}{|c|}{ Idioma } \\
\hline Português & 3 & 30 \\
\hline Inglês & 7 & 70 \\
\hline \multicolumn{3}{|c|}{ País de origem } \\
\hline Brasil & 3 & 30 \\
\hline EUA & 1 & 10 \\
\hline Itália & 1 & 10 \\
\hline Austrália & 1 & 10 \\
\hline Nova Zelândia & 1 & 10 \\
\hline Reino Unido & 1 & 10 \\
\hline Chile & 1 & 10 \\
\hline Canadá & 1 & 10 \\
\hline \multicolumn{3}{|c|}{ Bases de dados } \\
\hline BDENF & 1 & 10 \\
\hline LILACS & 1 & 10 \\
\hline SCIELO & 2 & 20 \\
\hline PUBMED & 6 & 60 \\
\hline
\end{tabular}




\section{Estratégias utilizadas para encaminhamento aos cuidados paliativos}

Quando o tratamento focado na cura deixa de ser interessante para o paciente a transição do cuidado para uma abordagem paliativa exclusiva se faz essencial para promover qualidade de vida ao indivíduo. Um dos pontos dificultadores para o direcionamento do paciente para o cuidado paliativo em neopediatria é saber quando o suporte invasivo deixa de ser um aliado para tentar sustentar a vida e se torna mais um sofrimento para criança, por isso médicos e enfermeiros utilizam ferramentas que consigam estratificar o paciente e sua demanda de cuidado (Bowlby, 2015).

Além de avaliar critérios como prognóstico e manejo de sintomas, escalas e checklists se mostram como instrumentos que otimizam o processo da tomada de decisão para encaminhar o paciente para o cuidado paliativo. Com esses instrumentos os profissionais avaliam a necessidade de cuidado, o benefício que o paciente teria com os procedimentos que possam ser realizados e a qualidade de vida a curto e longo prazo. Outra estratégia utilizada é a corresponsabilização da família no cuidado, a equipe esclarece a condição do quadro do paciente, seu prognóstico e quais as alternativas podem ser tomadas, diante disso juntos optam ou não por redirecionar a criança para assistência paliativa (Locatelli et al., 2020).

\section{Manejo dos sintomas}

Um dos pontos principais destacados nas obras selecionadas durante a revisão da literatura foi o manejo dos sintomas das crianças em cuidados paliativos. A forma mais estratégica para promover melhor qualidade de vida aos pacientes internados em UTIs neopediátricas foi o controle das alterações sintomáticas que diminuem o conforto do paciente (Poles \& Bousso, 2004).

Avaliar os sintomas em pediatria e neonatologia é algo que gera certa dificuldade, devido às barreiras de comunicação existentes, principalmente no manejo da dor em neonatos e lactentes. A conduta adotada muitas vezes é baseada no empirismo dos profissionais, mas com um conhecimento acerca da clínica de cada paciente é possível avaliar a necessidade de analgesia, sedação ou até mesmo medidas não farmacológicas para aliviar a dor. Uma das formas relatadas para o manejo da dor e controle da temperatura corporal de pacientes internados em unidades de terapia intensiva neopediátrica é a adoção do contato pele a pele durante a realização de procedimentos ou durante alguns momentos do dia, o aconchego proporcionado pela técnica reduz os níveis de estresse da criança e melhora até mesmo seus parâmetros homeostáticos (Callahan et al., 2019).

A dificuldade no manejo dos sintomas diminui consideravelmente quando existem protocolos e escalas que facilitam a visualização da demanda de cada criança. Para profissionais com pouca experiência essas ferramentas funcionam como um guia que ajuda a identificar a demanda para possíveis intervenções. Em serviços que não há equipe multidisciplinar de cuidados paliativos ofertar essas estratégias permite que pelo menos haja um facilitador para que o manejo dos sintomas seja feito da forma mais adequada (Marçola et al., 2017).

\section{Implementação de uma equipe multidisciplinar e a abordagem holística do paciente}

Durante a leitura dos artigos foi observado que existem barreiras para implementação de uma equipe multidisciplinar em unidades de terapia intensiva neonatal como por exemplo formação, fatores ambientais de falta de privacidade e isolamento, luto, perda de equipe e até mesmo prática de procedimentos. Essas são as barreiras mais evidentes para o cuidado holístico ao paciente e seus familiares (Ng et al., 2018).

A equipe médica e de enfermagem são as que mais atuam diretamente com esses pacientes, porém não é o suficiente para uma abordagem biopsicossocial pois o cuidado paliativo vai além da assistência focada na patologia e exige capacitação de uma equipe multidisciplinar (Kilcullen \& Ireland, 2017).

Dado os tópicos Maria do Carmo Vicensi descreve: 
Determinada a necessidade dos cuidados paliativos, a equipe multiprofissional da unidade deve entrar em cena com a execução de atividades assistenciais que não se restrinjam apenas às ações mecanicistas, ou seja, devem ser realizadas intervenções ligadas tanto a aspectos higiênicos, estéticos, de conforto físico e de minimização da dor, quanto a assistência psicológica, espiritual e social, gerando assim uma aproximação maior entre o paciente, familiares e os profissionais inseridos no tratamento (Vicensi, 2016).

Observa- se que a presença de uma equipe multidisciplinar e o desenvolvimento de protocolos específicos para os cuidados paliativos permitem uma assistência mais efetiva e facilita o trabalho dos profissionais. A implementação precoce de intervenções paliativas melhora a qualidade de vida do paciente (Callahan et al., 2019; Llédo-Morera, 2020).

\section{Abordagem psicoespiritual dos familiares e sua importância no processo dos cuidados paliativos pediátricos.}

A definição de espiritualidade é a busca do significado da vida, é a relação ou a experiência de conexão consigo mesmo, é o significado do sagrado. Esta definição também se aplica à pediatria. O cuidado paliativo pediátrico é definido como o cuidado ativo e total do corpo, mente e espírito da criança e do adolescente que tem uma doença grave e ameaçadora de vida, envolvendo também o suporte aos familiares (Kilcullen \& Ireland, 2017; Verri et al., 2019).

Com os avanços na tecnologia de sustentação da vida de uma criança gravemente enferma, as famílias passam a conviver com dilemas na tomada de decisões. Em um curto espaço de tempo, a família se depara com o cansaço físico e emocional, fazendo com que apareça por vezes, dúvidas e até a descrença de uma boa evolução no quadro do paciente (Marlow et al., 2021).

Contudo, eles ainda são responsáveis por aprender sobre a condição do filho, informar-se sobre as opções de tratamento e avaliar os riscos e benefícios em conjunto com os profissionais. Na maioria das vezes, as famílias buscam apoio na religião ou na vivência da espiritualidade para este momento delicado, como fonte de conforto e esperança.

\section{Vínculo afetivo e sua importância para a diminuição do estresse da criança}

Os seres humanos se desenvolvem através das interações uns com os outros por serem relacionais e dependentes. Desde o nascimento, o bebê precisa do auxílio de um adulto para se desenvolver e é este adulto que será responsável por sua nutrição e oferecerá a atenção e o carinho necessários para que essa criança possa se sentir segura e estimulada a evoluir em relação aos aspectos físicos, cognitivos e emocionais (Locatelli et al., 2020).

No cuidado paliativo o vínculo vem para colaborar no desenvolvimento de conforto tanto para o paciente, como para a sua família, diminuindo as taxas de depressão familiar, ansiedade e angústia, apresentando também uma melhora no quadro do paciente. A construção de um vínculo afetivo deixa as pessoas mais confiantes e preparadas para lidar com a questão “final da vida".

A partir da Teoria do Apego, desenvolvida por John Bowlby (2015), evidencia-se o papel essencial da formação de uma base segura, a partir da qual as demandas principais do indivíduo no começo da vida podem ser supridas. Ao se deparar com uma situação ameaçadora, nosso estilo de apego será acionado como uma tentativa de lidar com o evento buscando previsibilidade e familiaridade. O apego, pode ser compreendido aqui como uma força relacional que será ativada durante todo o ciclo vital. Quando o apego seguro se efetiva, os mecanismos de enfrentamento de situações adversas demonstram sua grande representatividade. A elaboração psíquica da construção de vínculos e seus possíveis rompimentos, é uma temática persistente e transversal na presença de uma doença ameaçadora da vida. O sofrimento inerente a situação, pode ser expresso em uma condição de autenticidade e proximidade afetiva que ultrapassa barreiras protocolares e possibilita que a vivência seja única para os sujeitos envolvidos, cercada de qualidade de presença e cuidados que permitam uma significação para a pessoa 
doente e para seus familiares. Este espaço tende a auxiliar na construção da história vivida, suas lembranças, legado e na elaboração do luto.

\section{Considerações Finais}

Diversos obstáculos afetam diretamente a assistência como a formação, a complexidade dos procedimentos, a falta privacidade com paciente e familiares, despreparo profissional, dentre outros. Há uma carência de estudos que demonstram a vivência da equipe multidisciplinar que atua com CPs em unidades de terapia intensiva, sendo assim percebe-se a necessidade de pesquisas que sinalizem a prática dos profissionais envolvidos nos $\mathrm{CP}$, a capacitação dos mesmos para atuarem com qualidade, melhor organização do serviço e uma assistência adequada.

Por fim, conclui-se que a revisão de literatura conseguiu reunir um conteúdo consistente e muito proveitoso, contribuindo para o enriquecimento do conhecimento acerca dos Cuidados Paliativos e como os profissionais médicos e da enfermagem conseguem abordar cuidados paliativos na uti neonatal buscando sempre oferecer a melhor assistência a seus pacientes e familiares apesar de todos os desafios encontrados na rotina do serviço.

Para trabalhos futuros, espera-se estudos com foco nas estratégias de Cuidados Paliativos, principalmente em pacientes pediátricos e neopediátricos, para que mais profissionais tenham acesso a informações sobre o tema para estarem melhor preparados para atender esses pacientes e seus familiares.

\section{Referências}

Almeida, F. A., Moraes, M. S., \& Cunha, M. L. R. (2016). Taking care of the newborn dying and their families: Nurses' experiences of neonatal intensive care. Revista da Escola de Enfermagem da Usp, 122-129.

Baldissarella, L., \& Dell'Aglio, D. D. (2009). No limite entre a vida e a morte um estudo de caso sobre a relação pais/bebê em uma UTI Neonatal. Estilos Clínicos, 14(26), 68-89.

Bowlby, J. (2015). Formação e rompimento dos laços afetivos.

Callahan, K., Steinwurtzel, R., Brumarie, L., Schechter, S. \& Parravicini, E. (2019). Early palliative care reduces stress in parents of neonates with congenital heart disease: validation of the "Baby, Attachment, Comfort Interventions". Journal Perinatol, 1640-1647.

Côté, A. J., Payot, A., \& Gaucher, N. (2020). Paediatric palliative care in practice: Perspectives between acute and long-term healthcare teams. Acta Paediatrica, 109(3), 613-619.

Hhnner, N., \& Boss, R. D. (2017). Neonatologist training in communication and palliative care. Seminars In Perinatology, 41(2), 106-110.

Kilcullen M., \& Ireland, S. (2017). Palliative care in the neonatal unit: neonatal nursing staff perceptions of facilitators and barriers in a regional tertiary nursery. BMC Palliaivet Care, 16(1). DOI: 10.1186/s12904-017-0202-3.

Lledó-Morera, À., \& Bosch-Alcaraz, A. (2020). Analysis of how nurses cope facing the death of a paediatric patient. Enfermagem Intensiva. DOI: 10.1016/j.enfi.2020.03.004. Epub ahead of print. PMID: 32855043.

Locatelli, C., Corvaglia, L., Simonazzi, G., Bisulli, M., Paolini, L., \& Faldella, G. (2020). "Percorso giacomo": an italian innovative service of perinatal palliative care. Front Pediatr.

Marcola, L., Barbosa, S. M. M., Zoboli, I., Polastrini, R. T. V. \& Ceccon, M. E. J. (2017). Análise dos óbitos e cuidados paliativos em uma unidade de terapia intensiva neonatal. Revista. Paulista de pediatria, 35(2), 125-129.

Marlow, N., Shaw, C., Connabeer, K., Aladangady, N., Gallagher, K., \& Drew, P. (2021). End-of-life decisions in neonatal care: a conversation analytical study. Arch Dis Child Fetal Neonatal, 106(2), 184-188.

Ng, S. K. F., Keenan, N., Swart, S. \& Berry, M. J. (2018). Palliative care in a tertiary neonatal intensive care unit: a 10-year review. BMJ Supportive \& Palliative Care. DOI: 10.1136/bmjspcare-2018-001538

Poles, K., \& Bousso, R. S. (2004). Enfermeira e a família no processo de morte da criança: evidências do conhecimento. Revista Sociedade Brasileira de Enfermagem Pediátrica, 4(1), 11-18.

Santos, CMDC, Pimenta, CADM, \& Nobre, MRC (2007). A estratégia PICO para a construção da pergunta de pesquisa e busca de evidências. Revista LatinoAmericana de Enfermagem , 15, 508-511. 
Research, Society and Development, v. 10, n. 10, e506101018277, 2021

(CC BY 4.0) | ISSN 2525-3409 | DOI: http://dx.doi.org/10.33448/rsd-v10i10.18277

Schwartz, T. M., \& Chalupka, S. (2019). Pediatric Palliative Care: Where Are We Now? Pediatric Nursing: Jannetti Publications, 45(5), 224-230.

Silva, I. N., Salim, N. R., Szylit, R., Sampaio, P. S. S., Ichikawa, C. R. F., \& Santos, M. R. (2017). Knowing nursing team care practices in relation to newborns in end-of-life situations. Escola Anna Nery. GN1 Genesis Network, 21( 4), 1-7.

Silva, L. C. S. P., Valença, C. N., \& Germano, R. M. (2010). Estudo fenomenológico sobre a vivência da morte em uma unidade de terapia intensiva neonatal. Revista Brasileira de Enfermagem, 63(5), 770-774.

Souza, M. T. D., Silva, M. D. D., \& Carvalho, R. D. (2010). Revisão integrativa: o que é e como fazer. Einstein (São Paulo), 8, 102-106.

Sociedade Brasileira de Pediatria (Brasil). (2017). Cuidados Paliativos Pediátricos: O que são e qual sua importância? Cuidando da criança em todos os momentos. Departamento Científico de Medicina da Dor e Cuidados Paliativos.

Verri, E. R., Bitencourt, N. A. S., Oliveira, J. A. S., Santos Junior, R., Marques, H. S., Porto, M. A., \& Rodrigues, D. G. (2019). Profissionais de enfermagem: compreensão sobre cuidados paliativos pediátricos. Revista de Enfermagem Ufpe On Line, 13(1), 126-136.

Vicensi, M. C. (2016). Reflexão sobre a morte e o morrer na UTI: a perspectiva do profissional. Revista Bioética, 24(1), 64-72.

World Health Organization. (2017). Palliative care.

World Health Organization. (2018). Integrating palliative care and symptom relief into paediatrics: a WHO guide for health care planners, implementers and managers.

Zimmermann, K., Cignacco, E., Engberg, S., Ramelet, A., Weid, N. V. D., Eskola, K., Bergstraesser, E., et al. (2018). Patterns of paediatric end-of-life care: a chart review across different care settings in Switzerland. Bmc Pediatrics, Springer Nature. DOI: http://dx.doi.org/10.1186/s12887-018-1021-2. 